\title{
SQSTM1/p62 in Intrahepatic Cholangiocarcinoma Promotes Tumor Progression Via Epithelial- Mesenchymal Transition and Mitochondrial Function Maintenance
}

Jiafeng Chen

Zhongshan Hospital Fudan University

Zheng Gao

Zhongshan Hospital Fudan University

Xiaogang Li

Zhongshan Hospital Fudan University

Yinghong Shi

Zhongshan Hospital Fudan University

Zheng Tang

Zhongshan Hospital Fudan University

Weiren Liu

Zhongshan Hospital Fudan University

Xin Zhang

Zhongshan Hospital Fudan University

Ao Huang

Zhongshan Hospital Fudan University

Qiang Gao

Zhongshan Hospital Fudan University

Guangyu Ding

Zhongshan Hospital Fudan University

Kang Song

Zhongshan Hospital Fudan University

Xiaoying Wang

Zhongshan Hospital Fudan University

Jian Zhou

Zhongshan Hospital Fudan University

Jia Fan

Zhongshan Hospital Fudan University

Xiutao Fu

Zhongshan Hospital Fudan University 


\section{ZhenBin Ding ( $\nabla$ ding.zhenbin@zs-hospital.sh.cn )}

Zhongshan Hospital Fudan University https://orcid.org/0000-0001-6613-8270

\section{Primary research}

Keywords: Intrahepatic cholangiocarcinoma, SQSTM1/p62, EMT, Metastasis, Mitophagy

Posted Date: October 18th, 2021

DOl: https://doi.org/10.21203/rs.3.rs-964992/v1

License: (c) (1) This work is licensed under a Creative Commons Attribution 4.0 International License. Read Full License 


\section{Abstract}

Background: SQSTM1/p62, as a selective autophagy receptor, regulates multiple signaling pathways participating in the initiation and progression of tumors. Since metastasis is still a main cause for intrahepatic cholangiocarcinoma (ICC)-associated mortality, this study aimed to explore the mechanism of p62 promoting progression of ICC.

Methods: Western blotting and immunohistochemical analysis were conducted to detect the expression level of protein p62 in ICC tissues. Subsequently, loss of function experiments was applied to define the role of p62 in the progression of ICC in vitro and in vivo. Mitochondrial function and mitophagy was evaluated by measuring oxygen consumption rates (OCR) and immunofluorescence detection respectively.

Results: Here we identified expression of p62 was significantly upregulated in ICC specimens compared to normal tissue. And we further illustrated that p62 expression was positively correlated with lymph-node metastasis and poor prognosis. Loss of function assays revealed that p62 not only promoted ICC cells proliferation, migration and invasive capacity in vitro, but also induced lung metastasis in xenograft mouse model. Mechanistically, high expression of p62 induced epithelial-mesenchymal transition (EMT) with upregulation of Snail1, Vimentin and down-regulation of E-Cadherin. Moreover, OCR assays and immunofluorescence cell staining demonstrated that the autophagy-dependent function of p62 may play a vital role in maintaining mitochondrial function of ICC by mitophagy.

Conclusions: These data provide new evidence and feasible mechanism that abundant p62 expression promote ICC progression, suggesting a promising therapeutic target for anti-metastatic strategies in ICC patients.

\section{Background}

Intrahepatic cholangiocarcinoma (ICC) refers to a kind of highly invasive hepatobiliary neoplasms arising in the intrahepatic biliary tree. Although considered a "rare" cancer in most countries, the incidence of ICC is rising in the recent decades which is responsible for $20 \%$ of liver-related deaths[1]. Moreover, due to the lack of identifiable factors and clinical symptoms, patients are frequently diagnosed at a locally advanced or metastatic stage with a poor 5-year survival rates ranging from 25 to $40 \%$ even after resection[2]. Unfortunately, the success of available chemotherapeutic regimens or few novel targeted agents is negligible, including the first-line therapy (gemcitabine and cisplatin). Therefore, there is an urgent necessity to understand the precise mechanism underlying ICC progression to identify novel therapeutic strategies.

Multifunctional adaptor protein SQSTM1/p62, the first selective autophagy receptor identified in metazoans, is found accumulated in various tumors, and indicated to act as an oncoprotein and promotor of tumor progression through Keap1-Nrf2, mTORC1, Caspase or NF-KB pathways[3-6]. 
However, the expression and underlying mechanism by which p62 influence ICC progression remain largely unexplored.

Epithelial-to-mesenchymal transition (EMT) and mitochondrial metabolic reprogramming are two different process to respond to the stress from tumor microenvironment (TME) which present an intricate correlation in progression of tumors. Intrahepatic invasion and distal metastasis of ICC need acquire migratory properties by a cell transdifferentiation process known as EMT during which epithelial cells adopt functional and structural characteristics of mesenchymal cells[7]. The most important transcription factors that drive EMT include SNAI1, TWIST1, ZEB1 as well as non-canonical transcription factors such as FOXC2 and GSC[8]. Mitochondria are central organelles in metabolic reprogramming which contribute to tumor initiation and progression. Cancer cells could meet the bioenergetic requirements through maintenance of mitochondria function[9].

Due to the importance of mitochondria, its homeostasis plays a vital role in fueling cancer initiation and progression which depends on the balance of degradation and biogenesis. Autophagy serves as an evolutionarily catabolic pathway through "self-digestion" of cytoplasmic components upon microenvironmental or therapeutic stress to maintain cell homeostasis[10]. In addition to macroautophagy, mitophagy which acts as the quality control of mitochondrial function is one form of selective autophagy that specifically recognize and degrade damaged mitochondria mediated by autophagy receptors, such as OPTN, NBR1, TAX1BP1 and SQSTM1[11, 12].

Accordingly, we assessed the expression of p62 in tumor and adjacent tissues of ICC. We further conduct in vivo and in vitro experiments to demonstrate that p62 was essential for EMT of ICC cells and p62 knockdown may impair EMT and mitochondria function, which results in inhibition of ICC growth and progression.

\section{Materials And Methods}

\section{Patients and specimens}

Tumor samples were achieved from 152 patients who underwent curative hepatectomy and were pathologically diagnosed with ICC at Liver Cancer Institute, Zhongshan Hospital, Shanghai, China. Of these, formalin-fixed paraffin-embedded specimens of 140 ICC patients were for tissue microarray (TMA) construction, and fresh samples of 12 patients collected for analysis of p62 expression between tumor and adjacent normal tissues. This study was approved by the ethics committee of Zhongshan Hospital and informed consent was obtained from each patient.

\section{Cell culture and transfection}

Human cholangiocarcinoma cell lines QBC939 and HCCC-9810 were purchased from the Cell Bank of Type Culture Collection of Chinese Academy of Sciences (Shanghai, China) and cultured in the RPMI1640 medium (HyClone, Logan, UT, USA) supplemented with 10\% fetal bovine serum (Gibco, USA). 
QBC939 and HCCC-9810 cells were transfected with lentivirus vectors encoding short hairpin RNA (shRNA) targeting p62 (shp62) or negative control vectors (shCtrl) (Genechem, shanghai, China) in the light of the manufacturer's directions, and stable cell lines were selected with $4 \mathrm{ug} / \mathrm{mL}$ puromycin. The shp62 coding sequences were 5'-CGTCAATAGCAACTGCTCCAA-3'. The efficiency of p62 knockdown was validated by western blotting.

\section{Western blotting analysis}

The total protein of whole cells or tissues were extracted using RIPA cell lysis buffer containing protease inhibitors (Beyotime, Shanghai, China). After determining protein concentrations using bicinchoninic acid (BCA) protein assay kit (Beyotime), equal amounts of proteins (20ug) were separated by SDS-PAGE and then transferred onto PVDF membranes. Following blocking in $5 \%(\mathrm{w} / \mathrm{v})$ non-fat milk, membranes were incubated with diluent primary and secondary antibodies. BeyoECL kit (Beyotime) was used for visualizing the protein bands. The following antibodies were utilized: anti-GAPDH (Abcam), anti-SQSTM1 (Abcam), anti-E-Cadherin (Cell Signaling Technology), anti-Vimentin (Abcam), anti-Snail (Abcam).

\section{Quantitative real-time reverse transcription polymerase chain reaction (qRT-PCR)}

Total RNA was extracted using Trizol reagent (Invitrogen, Carlsbad, CA, USA), and RNA was converted to cDNA using CDNA synthesis kit (YEASEN, Shanghai, China). qRT-PCR was performed using the SYBR Green Master MIX Kit (Tsingke, Shanghai, China) according to the manufacturer's instructions. Relative gene expression was evaluated by the $2^{-\triangle \Delta C t}$ method with GAPDH as an endogenous control. The primer sequences for RT-PCR are detailed as follows: GAPDH: forward: 5'-CATGGCCTTCCGTGTTCCTA-3', reverse: 5'-GCGGCACGTCAGATCCA-3'; p62: forward: 5'-GACTACGACTTGTGTAGCGTC-3', reverse:5'AGTGTCCGTGTTTCACCTTCC-3'; E-Cadherin: forward: 5'-GTAGGAAGGCACAGCCTGTC-3', reverse: 5'CAGCAAGAGCAGCAGAATCA-3'; Vimentin: forward: 5'-CTGCAGGACTCGGTGGACTT-3', reverse: 5'GAAGCGGTCATTCAGCTCCT-3'

\section{Cell migration and invasion assays}

To assess cell migration and invasion properties, the chamber was pre-coated with or without Matrigel

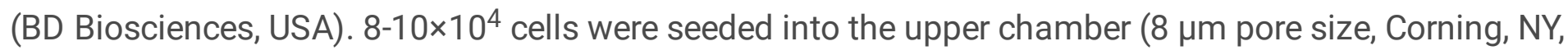
USA) with serum-free medium, and medium with $10 \%$ FBS was added to the lower chamber. After incubation for 24-48h, the migratory/invasive cells adhering to the lower surface were fixed with $4 \%$ paraformaldehyde and stained with crystal violet. The number of cells in the membrane were counted in 10 randomly selected visual fields under a microscope.

\section{Wound healing assay}

For wound healing assays, stable transfected and control cells were cultured in 6-well plates and were grown until reaching confluence. A sterile pipette was used to scratch a wound in the center of the cell monolayers. Images of the wounds were captured using a microscope at 0 and $24 \mathrm{~h}$. 


\section{Immunofluorescence cell staining}

Cells transfected with lentivirus-driven shp62 or shCtrl were seeded on sterile coverslips in a 12-well plate. After 24h, the cells were divided into two groups based on whether they were treated with iron chelator deferiprone (DFP). Then, the cells were fixed with $4 \%$ paraformaldehyde and permeabilized with $0.1 \%$ Triton X-100. Subsequently, the cells were incubated with primary and fluorescent secondary antibodies after blocking with $10 \%$ goat serum for 1 hour. The immunofluorescence signal was observed using Olympus FV-3000 confocal microscope (Olympus, Japan). The primary antibodies used were as follows: Anti-TOMM20 (1:500, Abcam); LC3B (1:50, Abcam); anti-E-Cadherin (1:200, Cell Signaling Technology); anti-Vimentin (1:200, Abcam). The secondary antibodies including Alexa 488-conjugated goat antirabbit/mouse IgG and Alexa 594-conjugated goat anti-rabbit/mouse IgG from Abcam were used.

\section{Assessment of mitochondrial respiration function}

Oxygen consumption rates (OCR) was determined by a Seahorse Extracellular Flux analyzer (Agilent, Santa Clara, CA, USA) using the Mito Stress Test Kit (Agilent). Briefly, $5 \times 10^{3}$ viable shp62 cells and their control cells were cultured in the XFe cell culture microplates overnight. Then the cell culture medium was replaced with DMEM XF base medium supplemented with $1 \mathrm{mM}$ pyruvate, $2 \mathrm{mM}$ glutamine and $10 \mathrm{mM}$ glucose and kept at $37^{\circ} \mathrm{C}$ for an additional one hour without $\mathrm{CO}_{2}$. OCR were measured prior to and after sequential injection of $1.5 \mu \mathrm{M}$ oligomycin, $0.5 \mu \mathrm{M}$ Carbonyl cyanide-4(trifluoromethoxy)phenylhydrazone (FCCP) and $0.5 \mu \mathrm{M}$ of rotenone plus antimycin A. All mitochondrial respiratory parameters were calculated and analyzed according to the manufacturer's instructions and protocols.

\section{Tissue microarray and immunohistochemistry staining}

TMA were constructed by Shanghai TUFEI Biotech Co. Ltd. (Shanghai, China). The anti-SQSTM1, anti-ECadherin, anti-Vimentin, anti-Snail antibodies were applied as primary antibodies in immunohistochemistry (IHC) based on a two-step protocol as previously described[13]. The staining intensity was categorized into levels $0,1,2$ and 3 . The percentage of positive staining cells was scored as $0(0 \%), 1(1-25 \%), 2(26-50 \%), 3(51-75 \%)$ and $4(76-100 \%)$. The product of intensity and percentage score was used as a final score.

\section{Animal xenograft models}

All animal experiments were approved by Ethics Committee of Zhongshan Hospital, Fudan University. 4week-old BALB/c nude male mice were purchased from SLAC Laboratory Animal Co. Ltd. (shanghai, China) and fed in specific-pathogen-free (SPF) condition. To evaluate the proliferation ability, $5 \times 10^{6}$ QBC939 cells stably transfected with lentivirus-driven shp62 or shCtrl were injected subcutaneously into the right flanks of nude mice. The mice were sacrificed about 4-6 weeks later when the size of tumor reached around $1000 \mathrm{~mm}^{3}$. The tumor tissue was minced into $2 \times 2 \times 2 \mathrm{~mm}^{3}$ slices and then subcutaneously implanted into flanks of other five 4-week-old nude mice each group. Tumor volumes were measured with an external caliper and calculated using the formula $\pi / 6 \times$ length $\times$ width $^{2}$ every five 
days as we previously described[13]. Forty days after subcutaneous injection, the mice were sacrificed by cervical dislocation, and the nodules on the liver and lung metastases were confirmed by HE staining.

\section{Statistical analysis}

Data are expressed as mean value \pm standard deviation (SD). Statistical significance for each variable was estimated by unpaired two-tailed Student's t test, Mann-Whitney $U$ test or one-way ANOVA test using GraphPad Prism 8.0, as appropriate. $\mathrm{p} \rrbracket 0.05$ was considered to indicate a statistically significant difference.

\section{Results}

\section{p62 is elevated in ICC tissues and associated with lymph-node metastasis and poor prognosis of ICC patients}

To identify the potential role of p62 in ICC, we compared the protein expression of p62 in 12 ICC tissue samples with adjacent nontumor tissues (Fig. 1A). A significant increase in p62 expression was observed in the tumor tissues compared with adjacent tissues $(P=0.0018)$. Among above tumor samples, 7 cases increased over two-fold, and 5 cases exhibited over one-fold increase (Fig. 1B). In addition, the results showed higher expression of protein p62 in tumor samples with lymph-node metastasis. $(P=0.0260)$ (Fig. 1C).

We further validated our hypothesis using TMA to evaluate the correlation between p62 and prognosis in 140 ICC patients who underwent curative liver resection. Notably, the p62 protein level in cytoplasm was higher in tumor tissues, especially in ICC with lymph-node metastasis (Fig. 1D). From the data quantified, only nine para-tumor samples had a IHC score of 3 or 4 points, while most of the tumor tissues had a score of more than 3 points, especially in ICC with lymph-node metastasis (Fig. 1E). Then, we divided the whole study cohort into p62 high expression (the IHC score $>4$ points; $n=41$ ) and p62 low expression (the $\mathrm{IHC}$ score $\leq 4$ points; $\mathrm{n=99}$ ) subgroup, and analyzed the relationship between the level of $\mathrm{p} 62$ expression and clinicopathological characteristics of ICC patients. The results indicated that older patients had higher expression of p62. However, there was no significant correlation between p62 expression and clinical characteristics such as gender, liver cirrhosis, tumor number, a-fetoprotein level, tumor capsule, tumor differentiation and vascular invasion (Supplemental Table 1). Moreover, high expression of p62 was significantly associated with lymph-node metastasis $(P=0.0346)$ (Fig. 1E). The heterogeneity of the degree of p62 expression in different status of lymph-node metastasis promoted us to examine whether p62 expression was associated with ICC patients' survival. Kaplan-Meier survival analysis revealed that ICC patients with high levels of $\mathrm{p} 62$ had a significantly shorter overall survival and higher cumulative recurrence rate than those with low expression of p62 ( $P=0.0082$ and $P=0.0034$, respectively) (Fig. 1F-G).

These observations indicated that high p62 expression in ICC not only contributes to lymph-node metastasis, but also influences patients' survival after liver resection. 
To further evaluate the cellular functions, especially metastasis potential, of p62 in vitro, we successfully constructed ICC cells with stably knockdown of p62 by shRNA, of which inhibitory efficiency was evaluated by western blotting (Fig. 2A). We investigated the role of p62 expression in cell proliferation and found that inhibition of $\mathrm{p} 62$ expression in ICC cells resulted in the decreased proliferation rate (Fig. 2B). Cellular migration is a characteristic of metastatic tumors and the first step of invasion. To assess whether p62 down-regulation in ICC cells affects cell migration, we performed transwell migration and wound-healing assays. Microscopic observation at 24 hours revealed a significant delay in the wound closure of p62-knockdown ICC cells compared with the control groups (Fig. 2E). As shown in Fig. 2C, silencing p62 expression was associated with a $33.8 \%$ and $47.9 \%$ reduction in migrated cells through transwell chamber in the HCCC-9810-shp62 and QBC939-shp62 cells compared with control cells, respectively. In addition, matrigel invasion assay was used to validate the invasive potentials of ICC cell lines. The cells with p62 knockdown showed a significant reduction in their ability to migrate through matrigel compared with control groups (Fig. 2D). Collectively, these in vitro assays suggested that knockdown of p62 expression attenuated proliferation, migration and invasion potentials of ICC cells.

\section{p62 inhibition impairs tumor growth and metastasis in vivo}

Next, we constructed a subcutaneous xenograft model using QBC939-shp62 and QBC939-shCtrl cells. Two weeks after inoculation, all mice successfully formed palpable tumors. Compared with controls, mice injected with QBC939-shCtrl cells showed significantly faster tumor growth which reflected by increased tumor size (Fig. 3A). After inoculation for 40 days, tumor volume of QBC939-shCtrl xenografts was $686.4 \pm 333.2 \mathrm{~mm}^{3}$, which was signi cantly larger than that derived from QBC939-shp62 group (207.3 $\pm 93.6 \mathrm{~mm}^{3}, P=0.0147$, Fig. 3B). Furthermore, metastatic analysis in vivo found that the downregulation of p62 reduced lung metastasis capacity in QBC939-shp62 group mice. In contrast, the lung metastasis rate was 80\% (4/5) in the QBC939-shCtrl group with more metastatic lung nodules (Fig. 3C-D). These results indicated that reduction of p62 expression could signilcantly inhibited tumor growth and progression of ICC in vivo.

\section{p62 promoted tumor progression of ICC cells through induction of EMT}

It has been reported that tumor cells mainly undergo epithelial-to-mesenchymal transitions to acquire the migratory and invasive properties. Therefore, we performed western blotting and immunofluorescence assays to determine the expression of EMT-related markers in ICC cells with different level of p62. The WB assay demonstrated a diminished expression of Snail and Vimentin, and increased expression levels of E-Cadherin in ICC cells after interference of p62 expression (Fig. 4A). qRT-PCR results also further confirmed that E-Cadherin mRNA was upregulated in ICC cells with p62 knockdown whereas Vimentin mRNA expression was significantly inhibited (Fig. 4B-C). Consistently, immunofluorescence assay also showed that down-regulation of p62 in ICC cells resulted in the increased expression of epithelial marker E-Cadherin and the decreased expression of mesenchymal marker Vimentin (Fig. 4D). We further used the 
IHC to investigate the relationship between expression of p62 and EMT-related markers in the 140 ICC tissues. Tumor samples expressing high p62 tended to have up-regulation of Vimentin and Snail while down-regulation of E-Cadherin (Fig. 4E). Taken together, these data implicated that the higher expression of p62 significantly may promote ICC tumor progression by inducing EMT process.

\section{Inhibition of p62 compromises mitochondrial function and mitophagy in ICC cells}

The mutual interplay between mitochondrial dysfunction and EMT in tumors has been recently highlighted, which is believed to be associated with progression to a metastatic and drug-resistant phenotype.[14] We investigated the functionality of mitochondria in ICC cells after inhibition of p62 expression. Measuring the mitochondrial OCR which quantify mitochondrial function more precisely demonstrated that basal and maximal respiratory capacity were significantly reduced in the p62knockdown ICC cells compared with control cells (Fig. 5A-C).

As is showed in the results above, inhibition of p62 caused mitochondria dysfunction. We hypothesized that p62 may be required for maintenance of mitochondrial respiration through mitophagy which could degrade dysfunctional mitochondrial. We then use the iron chelator deferiprone (DFP) triggered PINK1/Parkin-independent mitophagy and assessed early stage of mitophagy by visualizing colocalization of mitochondrial marker TOMM20 with the autophagosomal marker LC3. p62-knockdown ICC cells were found to have significant reduction of LC3 recruitment to mitochondria after DFP treatment (Fig. 5D). Together, these results suggested that inhibition of p62 impaired autophagic clearance of dysfunctional mitochondria, and disrupt mitochondrial homeostasis which may be associated with compromised metastatic potential of ICC.

\section{Discussion}

Mounting evidence identifies the selective autophagy receptor p62 is a multifunctional protein and involved in multiple signaling pathways that regulate inflammation, cancer and other common disease. And p62 expression is upregulated in a number of tumors, such as hepatocellular carcinoma, pancreatic cancer, breast cancer, but few articles reported that in $\operatorname{ICC}[6,15,16]$. p62 accumulation is involved in the arsenite-induced transformation of human hepatic epithelial (L-02) cells[17]. Likewise, our results showed there was higher p62 expression in ICC tissues compared with their normal tissues, suggesting p62 may be involved in formation of ICC. Moreover, p62 is not only overexpressed in early-stage of tumor, but also accumulated to promote tumor development[3, 4, 17-19]. Previous study has reported p62 can stabilize oncogenic transcription factor TWIST1 which contributes tumor cell proliferation and migration[20]. Consistent with that, our study found that p62 expression was notably higher in ICC tissues with lymphnode metastasis. Intriguingly, recent findings revealed p62 is a double-edged sword in cancer. For instance, p62 was essential for $\mathrm{H} 2 \mathrm{~A}$ ubiquitination through inhibiting E3 ligase RNF168s activity, which consequently impair DNA repair and increase the sensitivity of cancer cells to radiation[21]. In accordance, Flightless-I could hinder p62 dependent selective autophagy, and this was associated with 
accumulation of damage of protein and DNA which lead to tumorigenesis in breast cancer[22]. From a general perspective, these controversial roles of p62 may derived from the its cell-type dependent role of interactive hub in multiple signaling pathways.

In order to validate the positive association between p62 and tumor progression in ICC, we further revealed knockdown of p62 inhibited proliferation, migratory and invasive capabilities of ICC cells in vivo and tumor growth and metastasis in vitro. During the last decades, how cancer cells metastasize from the primary site has gained a lot of attention, one of which is a reversible dynamic process known as EMT. EMT endows epithelial cancer cells with enhanced motility and invasiveness, thus we conducted a series of experiments to validate the role of p62 in the EMT of ICC. Our data confirmed p62 downregulation remarkably decrease the expression of mesenchymal marker Vimentin, and increase epithelial marker E-Cadherin. Additionally, as a key transcription factor of EMT, Snail was also inhibited by p62 knockdown in ICC cell lines and tissues. Consistently, it has been reported that p62 upregulate Snail through the activation of nuclear factor kappa-light-chain-enhancer of activated B cells (NF-KB) to promote EMT[23]. In addition to this, as a selective autophagy receptor, p62 could promote EMT of Glioblastoma cells through targeting GSK-3 $\beta$ for degradation[24]. Given the ability of p62 to promote EMT of ICC, pharmacological target of p62 might represents feasible strategy with therapeutic potential.

Metabolic plasticity provides cancer cells the ability to cope with the changes of TME. Mitochondria, major source of ATP provision, are described as a vital player in tumor initiation and progression by reprogramming the bioenergetic cell metabolism[25]. The interplay between tumor progression and mitochondrial metabolism has been highlighted in the last decades. It has been widely observed that mitochondrial dysfunction was associated with increasing invasive, metastatic potential of cancer[26]. However, the precise mechanisms about how dysregulation of mitochondrial metabolism affect tumor progression are still a matter of debate. For this reason, we explore the effect of loss of p62 on mitochondrial metabolism and found that down-regulation of p62 impair mitochondrial function significantly in ICC cells.

Mitophagy is a special type of autophagy which mediates the clearance of dysfunctional or damaged mitochondria to alleviate cellular stress and maintain normal cell growth. It's indicated that mitophagy may play dichotomous roles in tumorigenesis and tumor progression[27, 28]. To mark mitochondria for mitophagy, ubiquitinated mitochondria need receptor proteins to integrate with autophagosomes, such as p62[12]. Meanwhile, it has been reported that besides PINK1/Parkin-dependent mitophagy, p62 can promote ubiquitination of mitochondrial independently of PINK1 and PRKN, and iron chelators could induce this kind of mitophagy[29, 30]. Consequently, we further verified whether p62 protein could regulate mitochondrial function by participating in mitophagy. Our data demonstrated that downregulation of p62 impair mitochondrial function and DFP-induced PINK1/Parkin-independent mitophagy simultaneously by utilizing mitochondrial respiration function test and immunofluorescence assay. We presumed that mitophagy may involve in mitochondrial regulation, thus facilitate migration and invasion of ICC cells. Similarly, previous study has revealed that loss of p62 impairs murine myeloid leukemia progression through significantly delaying removal of damaged mitochondria and impairing 
mitochondrial respiration[19]. Nonetheless, in the initiation of carcinogenesis, parkin-deficient mice are susceptible to spontaneous hepatocellular carcinoma which indicated that loss of mitophagy induces mitochondrial dysfunction and stimulates carcinogenesis[31]. Meanwhile, a growing number of articles have illustrated parkin or mitophagy play a suppression role in cancer[32]. These controversies may be ascribed to that mitophagy regulates EMT in multiple ways depending on tumor stage, TME, and metabolic reprogramming. In conclusion, it remains to be investigated which signaling pathway p62 involved participate in mitophagy to promote EMT in ICC.

\section{Conclusions}

In conclusion, we believe that p62 in intrahepatic cholangiocarcinoma can promote EMT and tumor progression, which may be caused by maintenance of mitochondrial function and mitophagy which degrades dysfunctional mitochondrial. Our results provide a novel insight that targeting for p62 is a potential prognostic and therapeutic candidate in intrahepatic cholangiocarcinoma.

\section{Declarations}

\section{Ethics approved and consent to participate}

This study was approved by the Ethics Committee of Zhongshan Hospital of Fudan University (registration number Y2019-271). All patients provided their written informed consent for data collection for research purposes.

\section{Consent for publication}

Not applicable.

\section{Availability of data and materials}

The datasets generated and analysed during the current study are available from the corresponding author on reasonable request.

\section{Competing interests}

The authors declare that they have no competing interests.

\section{Funding}

This work was supported by the National Natural Science Foundation of China (No. 81972229) and Natural Science Foundation of Shanghai (No. 20ZR1473100).

\section{Author's contributions}


ZBD, XTF and JFC conceived and designed the study. YHS, ZT provided research methods. JFC, ZG, XGL, WRL, XZ, AH, QG, GYD and KS participated in implementation of the study. JFC, ZG and XGL performed the statistical analysis. JFC, XYW, JZ, JF, XTF and ZBD drafted and revised the manuscript. All authors read and approved the final manuscript.

\section{Acknowledgements}

Not applicable.

\section{References}

1. Akinyemiju T, Abera S, Ahmed M, Alam N, Alemayohu MA, Allen C, Al-Raddadi R, Alvis-Guzman N, Amoako Y, Artaman A, et al. The Burden of Primary Liver Cancer and Underlying Etiologies From 1990 to 2015 at the Global, Regional, and National Level: Results From the Global Burden of Disease Study 2015. JAMA oncology. 2017;3(12):1683-91.

2. Mazzaferro V, Gorgen A, Roayaie S, Droz Dit Busset M, Sapisochin G. Liver resection and transplantation for intrahepatic cholangiocarcinoma. Journal of hepatology. 2020;72(2):364-77.

3. Saito T, Ichimura Y, Taguchi K, Suzuki T, Mizushima T, Takagi K, Hirose Y, Nagahashi M, Iso T, Fukutomi T, et al. p62/Sqstm1 promotes malignancy of HCV-positive hepatocellular carcinoma through Nrf2-dependent metabolic reprogramming. Nature communications. 2016;7:12030.

4. Zhang J, Yang S, Xu B, Wang T, Zheng Y, Liu F, Ren F, Jiang J, Shi H, Zou B, et al. p62 functions as an oncogene in colorectal cancer through inhibiting apoptosis and promoting cell proliferation by interacting with the vitamin D receptor. Cell proliferation. 2019;52(3):e12585.

5. Umemura A, He F, Taniguchi K, Nakagawa H, Yamachika S, Font-Burgada J, Zhong Z, Subramaniam S, Raghunandan S, Duran A, et al. p62, Upregulated during Preneoplasia, Induces Hepatocellular Carcinogenesis by Maintaining Survival of Stressed HCC-Initiating Cells. Cancer cell. 2016;29(6):935-48.

6. Hennig P, Fenini G, Di Filippo M, Karakaya T, Beer HD. The Pathways Underlying the Multiple Roles of p62 in Inflammation and Cancer. Biomedicines 2021, 9(7).

7. Thiery JP, Acloque H, Huang RY, Nieto MA. Epithelial-mesenchymal transitions in development and disease. Cell. 2009;139(5):871-90.

8. Puisieux A, Brabletz T, Caramel J. Oncogenic roles of EMT-inducing transcription factors. Nat Cell Biol. 2014;16(6):488-94.

9. Spinelli JB, Haigis MC. The multifaceted contributions of mitochondria to cellular metabolism. Nat Cell Biol. 2018;20(7):745-54.

10. Yu L, Chen Y, Tooze SA. Autophagy pathway: Cellular and molecular mechanisms. Autophagy. 2018;14(2):207-15.

11. Kirkin V. History of the Selective Autophagy Research: How Did It Begin and Where Does It Stand Today? Journal of molecular biology. 2020;432(1):3-27. 
12. Klionsky DJ, Abdelmohsen K, Abe A, Abedin MJ, Abeliovich H, Acevedo Arozena A, Adachi H, Adams $\mathrm{CM}$, Adams PD, Adeli K, et al: Guidelines for the use and interpretation of assays for monitoring autophagy (3rd edition). Autophagy 2016, 12(1):1-222.

13. Fu XT, Song K, Zhou J, Shi YH, Liu WR, Shi GM, Gao Q, Wang XY, Ding ZB, Fan J. Tumor-associated macrophages modulate resistance to oxaliplatin via inducing autophagy in hepatocellular carcinoma. Cancer cell international. 2019;19:71.

14. Guerra F, Guaragnella N, Arbini AA, Bucci C, Giannattasio S, Moro L. Mitochondrial Dysfunction: A Novel Potential Driver of Epithelial-to-Mesenchymal Transition in Cancer. Frontiers in oncology. 2017;7:295.

15. Aishima S, Fujita N, Mano Y, Iguchi T, Taketomi A, Maehara Y, Oda Y, Tsuneyoshi M. p62+ Hyaline inclusions in intrahepatic cholangiocarcinoma associated with viral hepatitis or alcoholic liver disease. Am J Clin Pathol. 2010;134(3):457-65.

16. Duran A, Hernandez ED, Reina-Campos M, Castilla EA, Subramaniam S, Raghunandan S, Roberts LR, Kisseleva T, Karin M, Diaz-Meco MT, et al. p62/SQSTM1 by Binding to Vitamin D Receptor Inhibits Hepatic Stellate Cell Activity, Fibrosis, and Liver Cancer. Cancer cell. 2016;30(4):595-609.

17. Liu X, Ling M, Chen C, Luo F, Yang P, Wang D, Chen X, Xu H, Xue J, Yang Q, et al. Impaired autophagic flux and p62-mediated EMT are involved in arsenite-induced transformation of L-02 cells. Toxicol Appl Pharmcol. 2017;334:75-87.

18. Karras P, Riveiro-Falkenbach E, Cañón E, Tejedo C, Calvo TG, Martínez-Herranz R, Alonso-Curbelo D, Cifdaloz M, Perez-Guijarro E, Gómez-López G, et al. p62/SQSTM1 Fuels Melanoma Progression by Opposing mRNA Decay of a Selective Set of Pro-metastatic Factors. Cancer cell. 2019;35(1):4663.e10.

19. Nguyen TD, Shaid S, Vakhrusheva O, Koschade SE, Klann K, Thölken M, Baker F, Zhang J, Oellerich T, Sürün D, et al. Loss of the selective autophagy receptor p62 impairs murine myeloid leukemia progression and mitophagy. Blood. 2019;133(2):168-79.

20. Qiang L, Zhao B, Ming M, Wang N, He TC, Hwang S, Thorburn A, He YY. Regulation of cell proliferation and migration by p62 through stabilization of Twist1. Proc Natl Acad Sci USA. 2014;111(25):9241-6.

21. Wang Y, Zhu WG, Zhao Y. Autophagy substrate SQSTM1/p62 regulates chromatin ubiquitination during the DNA damage response. Autophagy. 2017;13(1):212-3.

22. He JP, Hou PP, Chen QT, Wang WJ, Sun XY, Yang PB, Li YP, Yao LM, Li X, Jiang XD, et al. Flightless-I Blocks p62-Mediated Recognition of LC3 to Impede Selective Autophagy and Promote Breast Cancer Progression. Cancer Res. 2018;78(17):4853-64.

23. Wang Y, Xiong H, Liu D, Hill C, Ertay A, Li J, Zou Y, Miller P, White E, Downward J, et al. Autophagy inhibition specifically promotes epithelial-mesenchymal transition and invasion in RAS-mutated cancer cells. Autophagy. 2019;15(5):886-99.

24. Li H, Li J, Zhang G, Da Q, Chen L, Yu S, Zhou Q, Weng Z, Xin Z, Shi L, et al. HMGB1-Induced p62 Overexpression Promotes Snail-Mediated Epithelial-Mesenchymal Transition in Glioblastoma Cells 
via the Degradation of GSK-3ß. Theranostics. 2019;9(7):1909-22.

25. Moindjie H, Rodrigues-Ferreira S, Nahmias C. Mitochondrial Metabolism in Carcinogenesis and Cancer Therapy. Cancers 2021, 13(13).

26. Porporato PE, Payen VL, Pérez-Escuredo J, De Saedeleer CJ, Danhier P, Copetti T, Dhup S, Tardy M, Vazeille T, Bouzin C, et al. A mitochondrial switch promotes tumor metastasis. Cell reports. 2014;8(3):754-66.

27. Panigrahi DP, Praharaj PP, Bhol CS, Mahapatra KK, Patra S, Behera BP, Mishra SR, Bhutia SK. The emerging, multifaceted role of mitophagy in cancer and cancer therapeutics. Sem Cancer Biol. 2020;66:45-58.

28. Ray SK, Mukherjee S. Mitophagy in Carcinogenesis and Tumor progression- A New paradigm with Emerging Importance. Anti-cancer agents in medicinal chemistry 2021.

29. Yamada T, Dawson TM, Yanagawa T, lijima M, Sesaki H. SQSTM1/p62 promotes mitochondrial ubiquitination independently of PINK1 and PRKN/parkin in mitophagy. Autophagy. 2019;15(11):2012-8.

30. Allen GF, Toth R, James J, Ganley IG. Loss of iron triggers PINK1/Parkin-independent mitophagy. EMBO Rep. 2013;14(12):1127-35.

31. Fujiwara M, Marusawa H, Wang HQ, Iwai A, Ikeuchi K, Imai Y, Kataoka A, Nukina N, Takahashi R, Chiba T. Parkin as a tumor suppressor gene for hepatocellular carcinoma. Oncogene. 2008;27(46):6002-11.

32. Bernardini JP, Lazarou M, Dewson G. Parkin and mitophagy in cancer. Oncogene. 2017;36(10):131527.

\section{Figures}


(A)

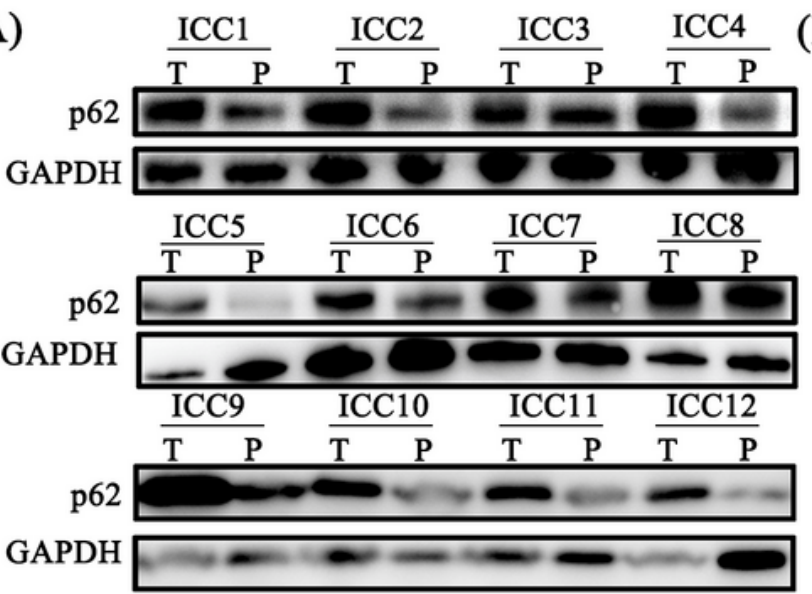

(D)

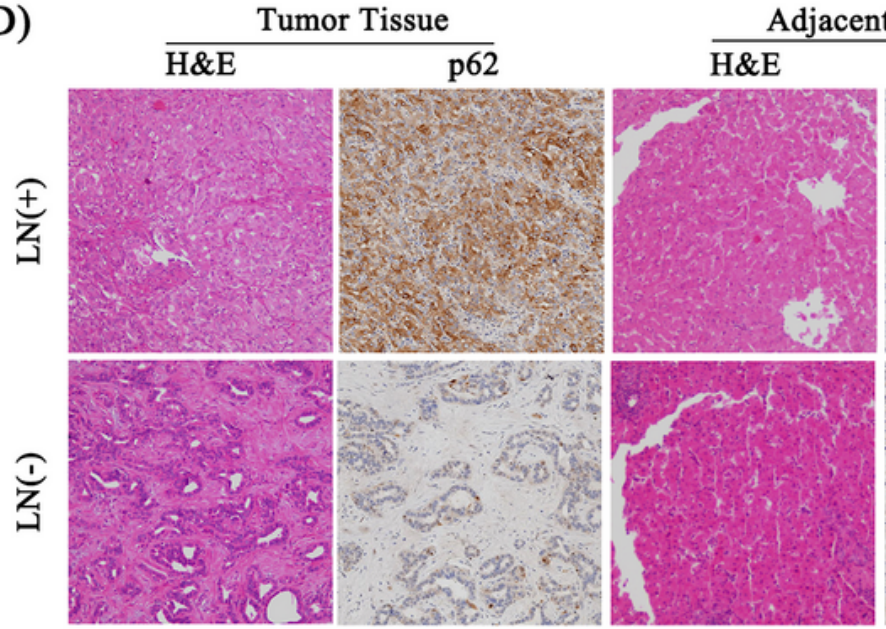

(F)

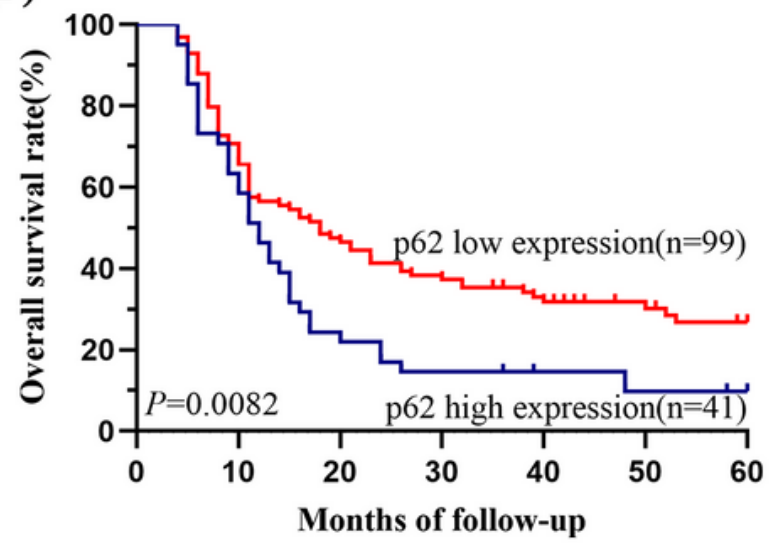

(C)

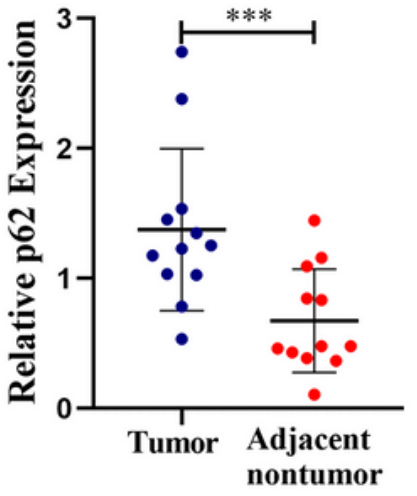

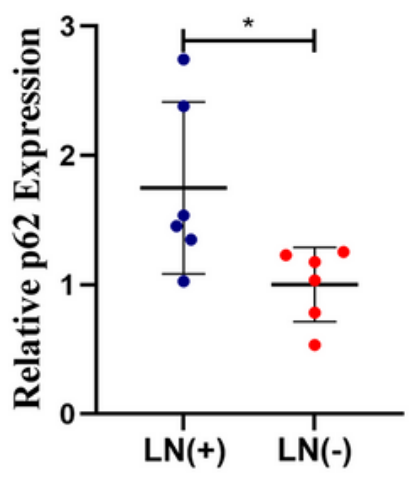

(E)

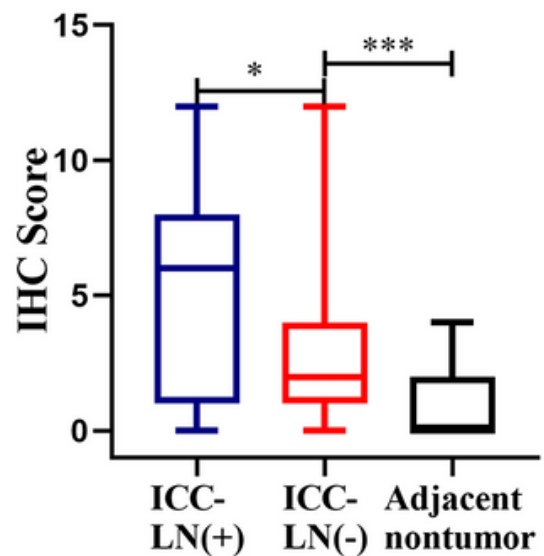

(G)

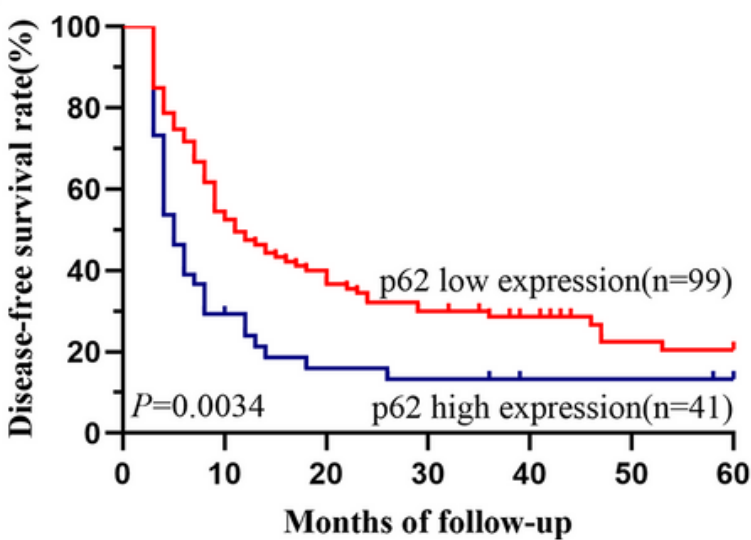

Figure 1

Expression of p62 in tissues of ICC with or without lymph-node (LN) metastasis and its effect on prognosis. (A) Western blotting images of p62 expression in 12 paired ICC and adjacent tissues with (ICC 7-12) or without LN metastasis (ICC 1-6). (B-C) Statistical analysis of p62 expression in 12 paired ICC and adjacent tissues. (D) Representative H\&E and immunohistochemistry staining (IHC) images of p62 expression in ICC and adjacent tissues from 140 patients. (E) Quantitative comparison of IHC scores 
between ICC and adjacent tissues with [LN (+), n=34] or without LN metastasis [LN (-), n=106]. (F-G) Kaplan-Meier showed overall survival time and disease-free survival time of 140 patients with different p62 expression. The data are presented as mean \pm SD or median (range). Statistical significance was assessed using Student's t test, log-rank test or Mann-Whitney U test, as appropriate. ${ }^{\star} p \otimes 0.05,{ }^{\star *} \mathrm{p} \llbracket 0.01$, $* * * \mathrm{p} \otimes 0.001$.
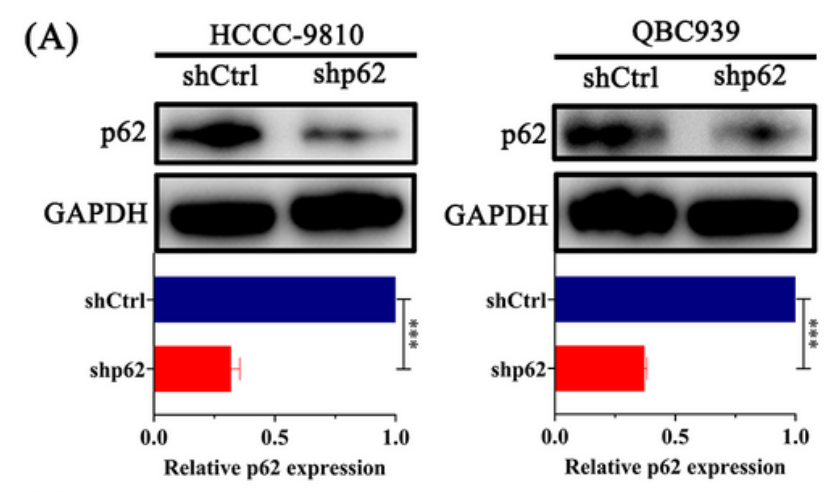

(B)

(C) Migration

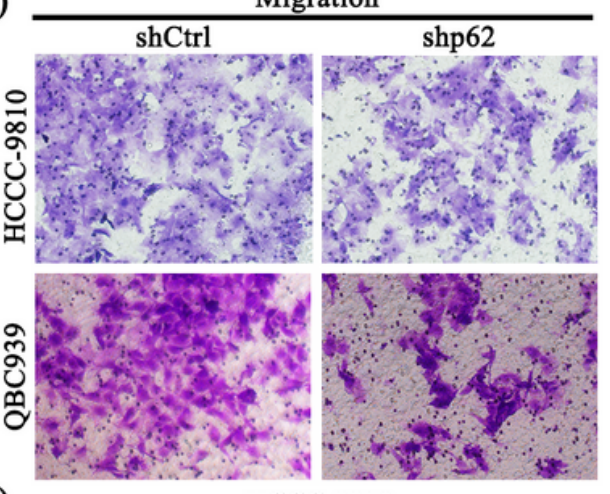

(E)
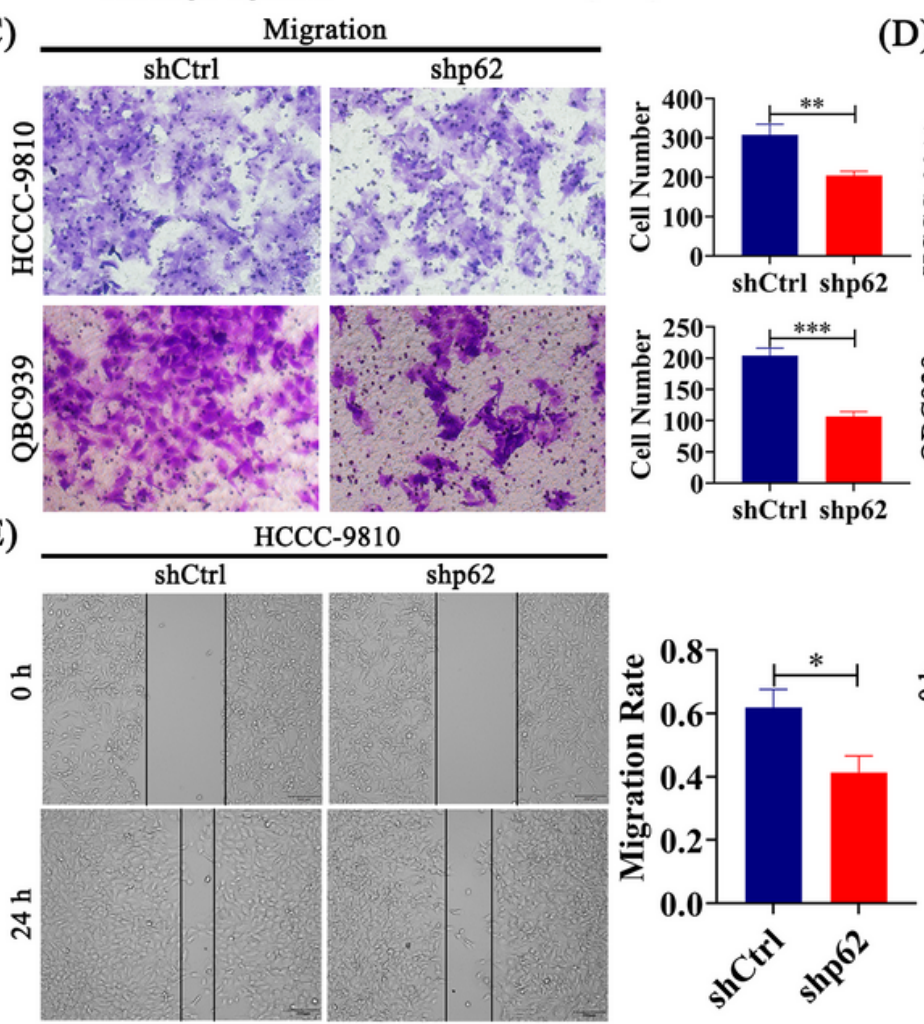

(D)
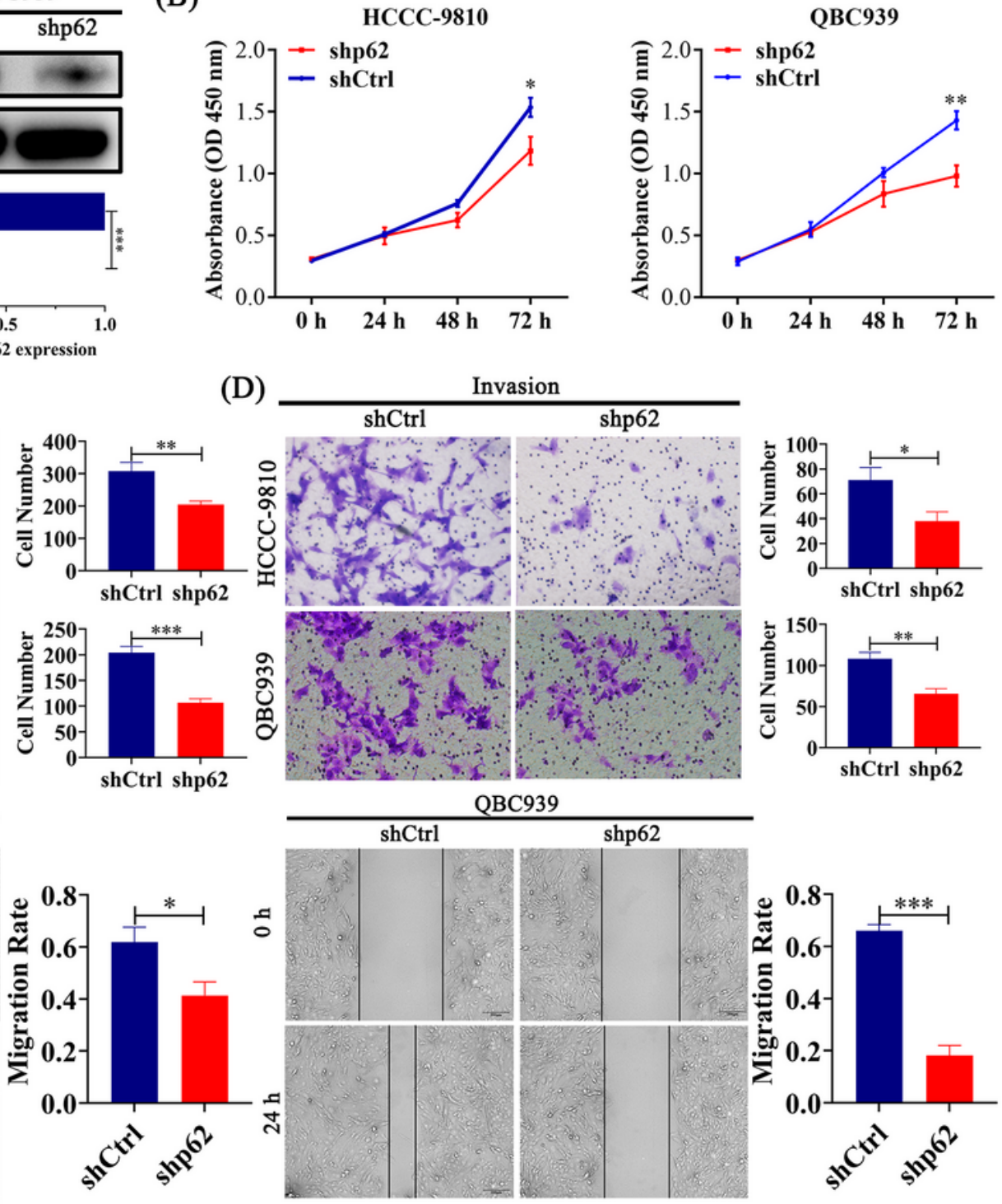

shp62

\section{Figure 2}

Inhibition of p62 impairs ICC progression in vitro. (A) Western blotting confirmation of p62 knockdown using shRNA in HCCC-9810 and QBC939 cells. The intensities of bands are quantified in the lower panels. (B) Quantification of cell proliferation of p62 knockdown (shp62) and control (shCtrl) HCCC-9810 and QBC939 cells by Cell Counting Kit-8 assay (CCK8). (C-D) Representative images and quantification of transwell migration assay and matrigel invasion assay of shp62 and shCtrl ICC cells. (E) Representative images of wound-healing assays, and histogram analysis of cell migration distance is showed in the 
right panels. The data are presented as mean $\pm S D$. Statistical significance was assessed using Student's $t$ test. *pख0.05, ${ }^{* *} \mathrm{p} \rrbracket 0.01, * * * \mathrm{p} \otimes 0.001$.

(A)

\section{QBC939 xenograft}

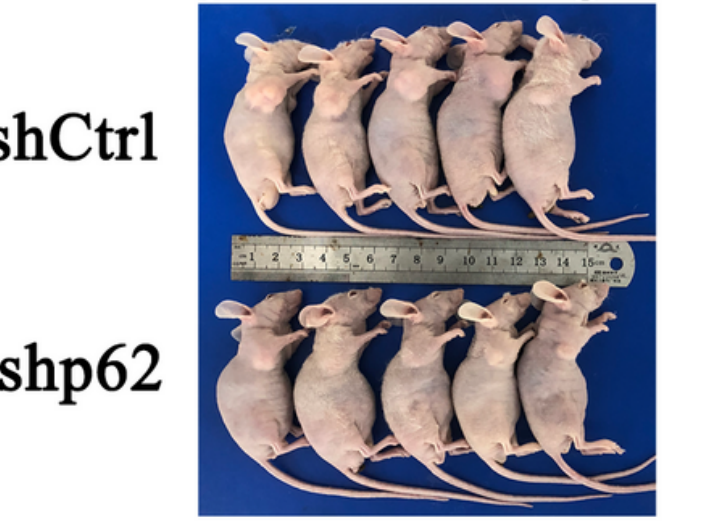

(B)

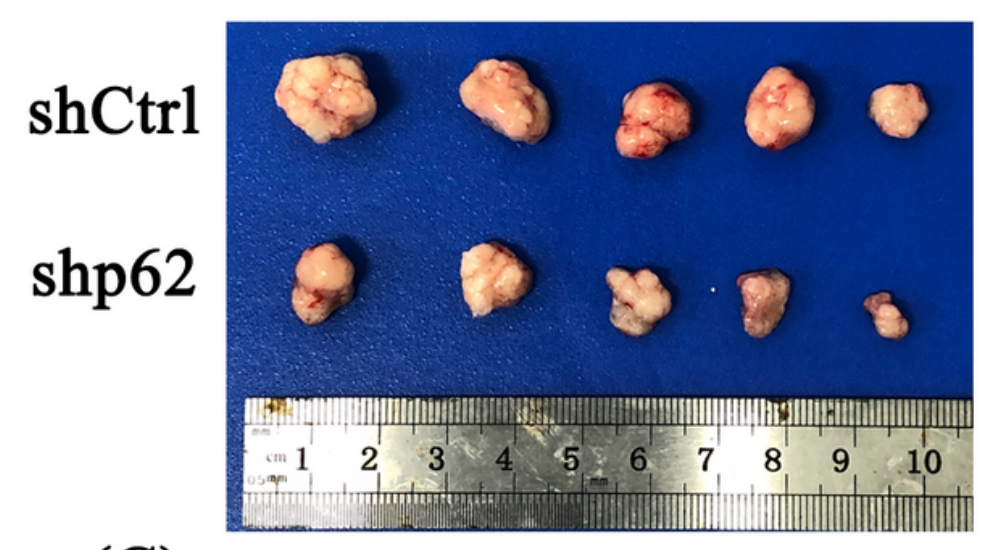

(C)
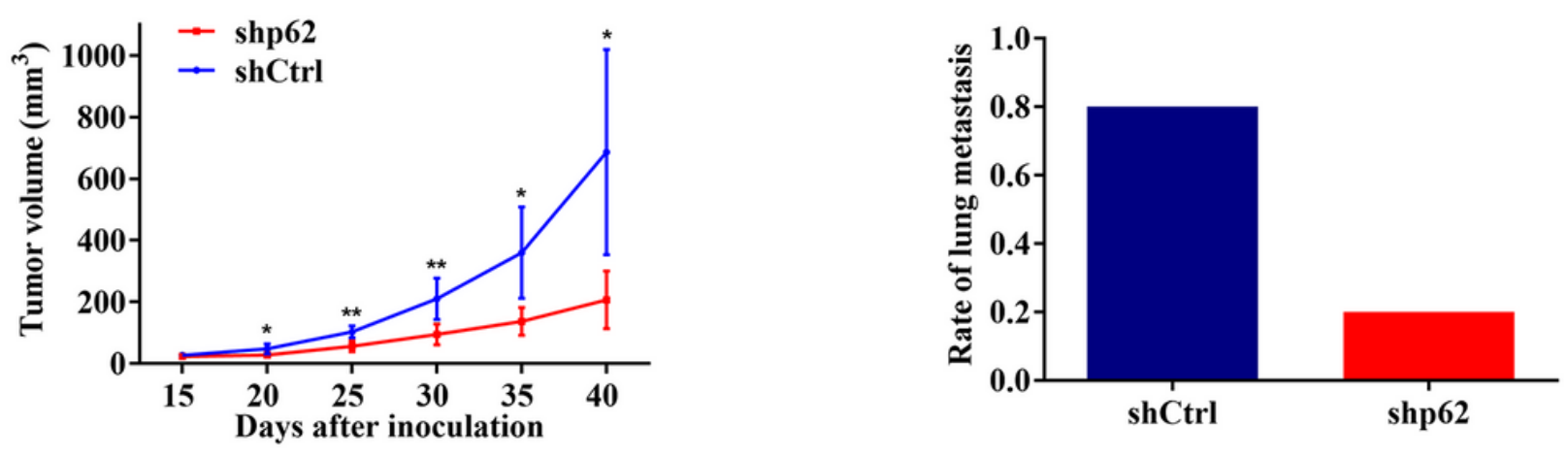

(D)
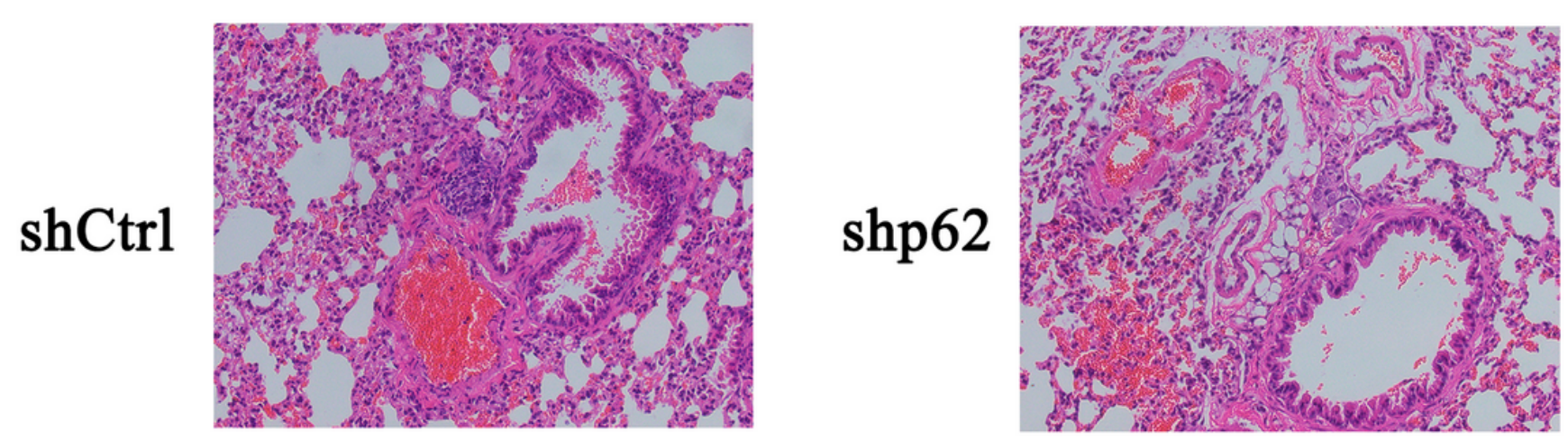

Figure 3

p62 promotes ICC growth and metastasis in vivo. (A) Overview of tumors in BALB/c mice subcutaneously implanted with QBC939-shp62 or QBC939-shCtrl cells ( $n=5 /$ group). (B) Comparison of tumor volumes in mice model implanted with QBC939-shp62 or QBC939-shCtrl cells. (C) Rate of lung metastasis in different groups. (D) Representative H\&E staining images of lung metastasis. The data are presented as mean $\pm S D$. Statistical significance was assessed using Student's t test. *p $\llbracket 0.05,{ }^{\star *} \mathrm{p} \rrbracket 0.01$. 


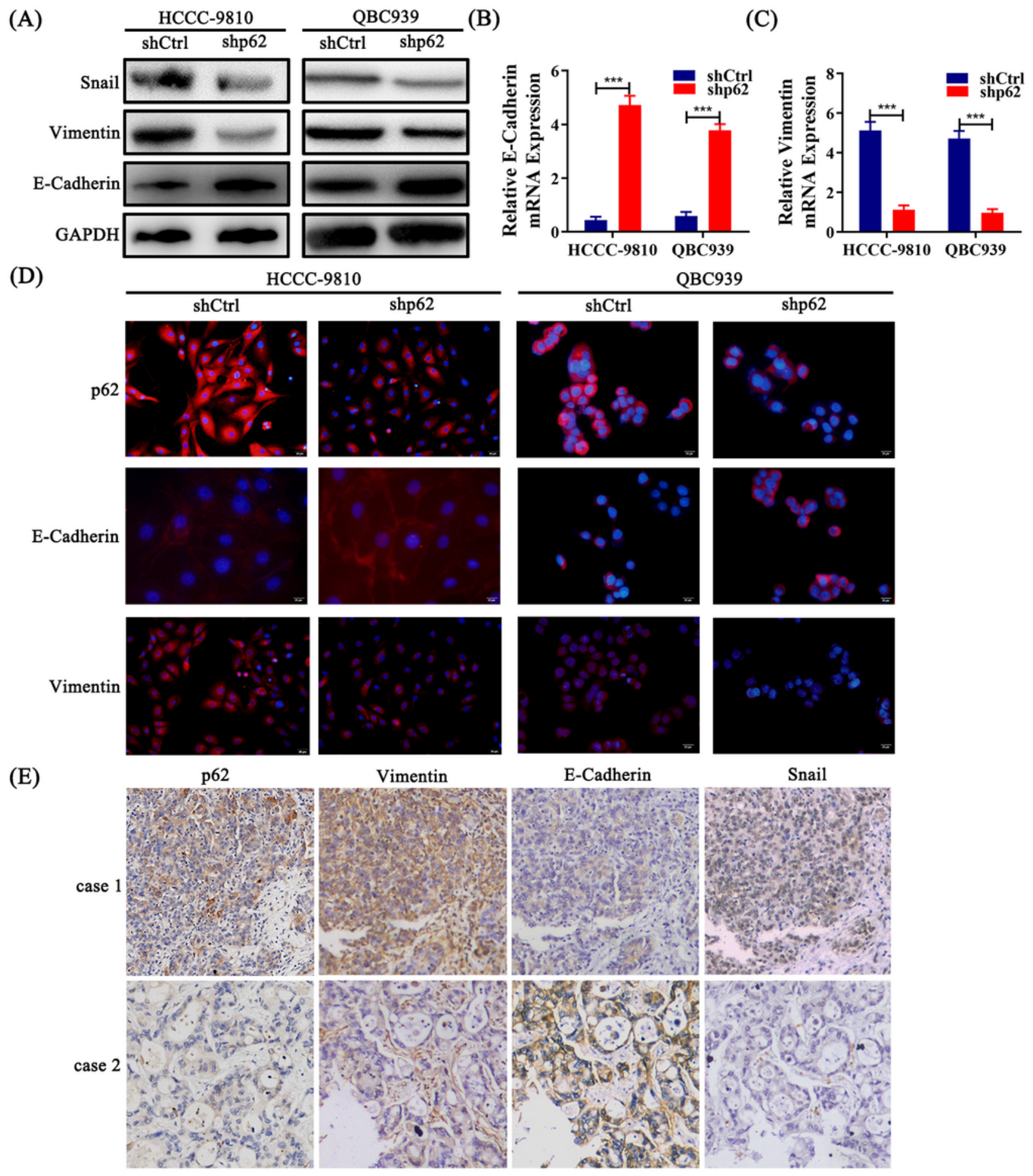

\section{Figure 4}

High level of p62 promoted tumor progression through induction of EMT. (A) Western blotting determined E-Cadherin, Vimentin, Snail proteins expression in p62 knockdown(shp62) and control (shCtrl) HCCC9810 and QBC939 cells. (B-C) qRT-PCR analysis of E-Cadherin and Vimentin mRNA levels in ICC cells with different p62 expression. (D) Immunofluorescent staining for p62, E-Cadherin and Vimentin of ICC cells with different p62 expression. DAPI stain was used to identify nuclei. (E) Representative images of levels 
of p62, E-Cadherin, Vimentin and Snail in ICC tissues detected by immunohistochemistry staining. The

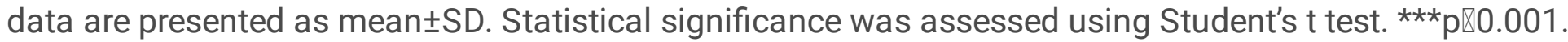

(A)

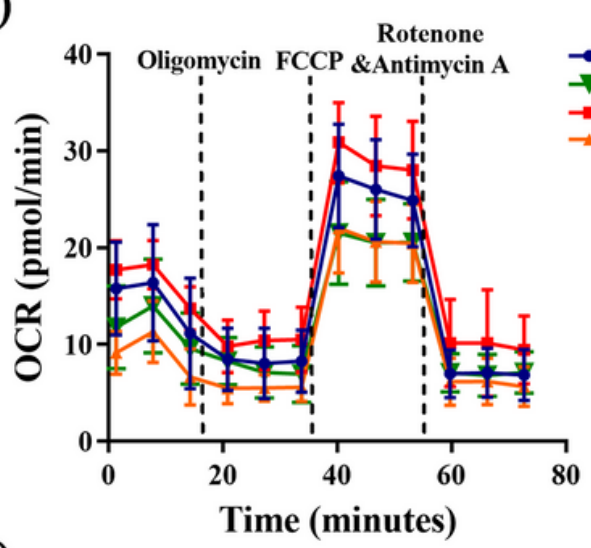

(D)

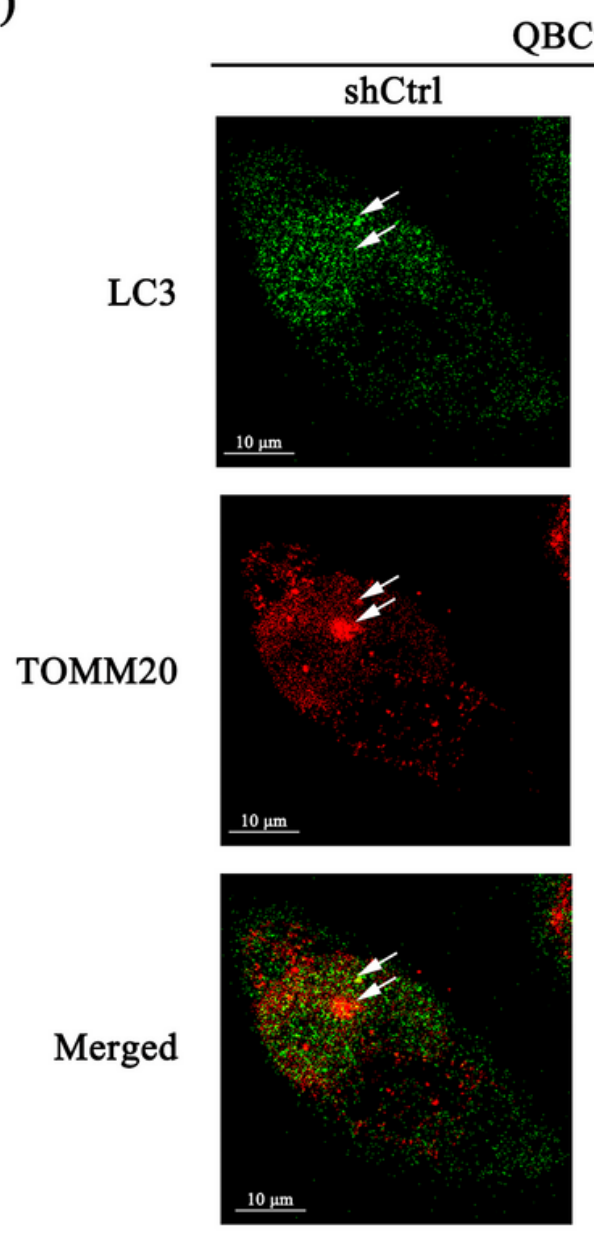

(B)
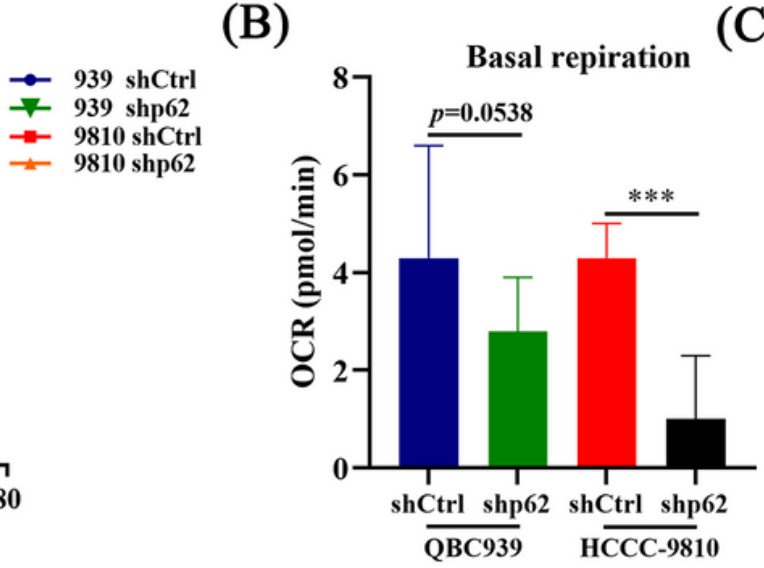

(C)

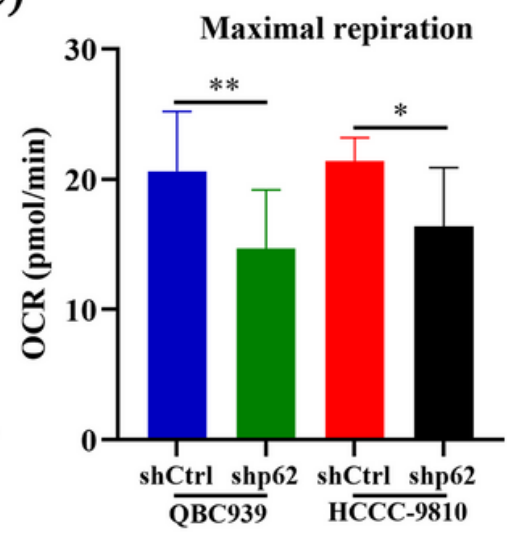

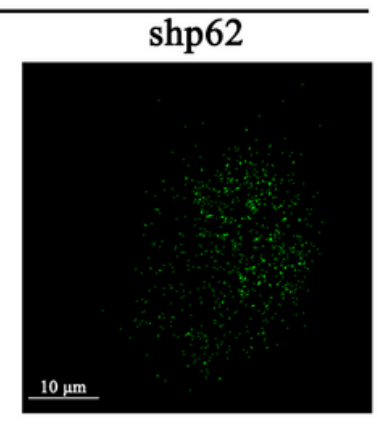
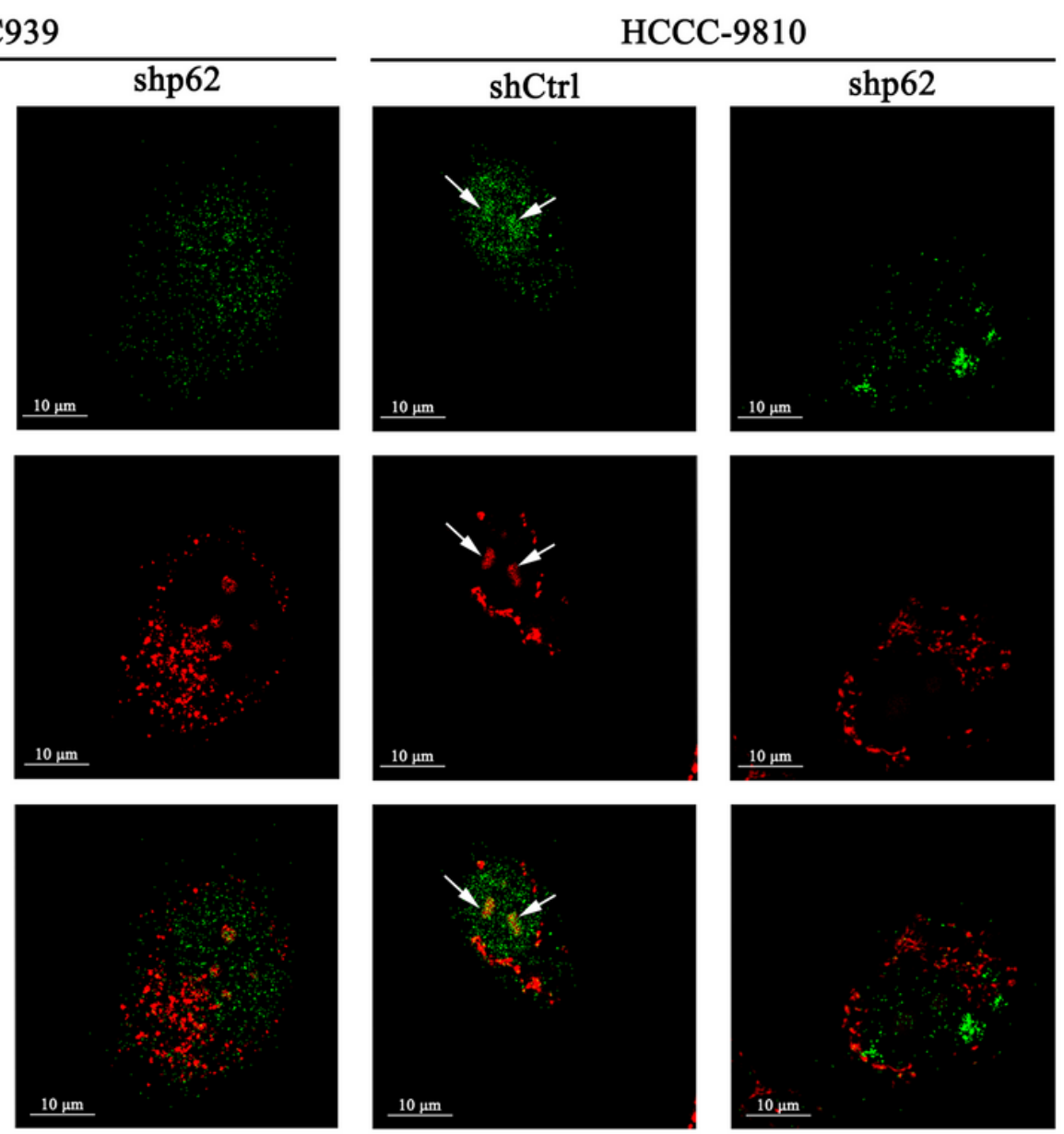

\section{Figure 5}

Inhibition of p62 compromises mitochondrial function and mitophagy in ICC cells. (A) Mitochondrial respiration of HCCC-9810-shp62 and QBC939-shp62 or HCCC-9810-shCtrl and QBC939-shCtrl cells was determined by measuring the oxygen consumption rate (OCR). (B-C) The rates of basal respiration, maximal respiration were compared between shp62 and shCtrl groups of HCCC-9810 and QBC939 cells. (D) Immunofluorescent staining for TOMM20 and LC3 of ICC cells with different p62 expression after 12h 
treatment with the iron chelator deferiprone (DFP). Arrows indicate colocalization of both markers. The data are presented as mean \pm SD. Statistical significance was assessed using Student's t test. ${ }^{\star} \mathrm{p} \otimes 0.05,{ }^{* *} \mathrm{p}$ $\varangle 0.01, * \star * \mathrm{p} \llbracket 0.001$.

\section{Supplementary Files}

This is a list of supplementary files associated with this preprint. Click to download.

- Supplementalmaterial.docx 